Rabaska

Revue d'ethnologie de l'Amérique française

\title{
Chaire de recherche McCain en ethnologie acadienne (CREMEA)
}

\section{Ronald Labelle}

Volume 5, 2007

URI : https://id.erudit.org/iderudit/019067ar

DOI : https://doi.org/10.7202/019067ar

Aller au sommaire du numéro

Éditeur(s)

Société québécoise d'ethnologie

ISSN

1703-7433 (imprimé)

1916-7350 (numérique)

Découvrir la revue

Citer ce document

Labelle, R. (2007). Chaire de recherche McCain en ethnologie acadienne (CREMEA). Rabaska, 5, 226-230. https://doi.org/10.7202/019067ar d'utilisation que vous pouvez consulter en ligne.

https://apropos.erudit.org/fr/usagers/politique-dutilisation/ 
C. Autres - Le titulaire de la Cofram a préparé les analyses et l'index des types des vingt-six contes du beau recueil de Joseph-Médard Carrière, une publication posthume préparée à l'Université de Sudbury : Contes du Détroit recueillis par Joseph-Médard Carrière, présentés par Marcel Bénéteau et Donald Deschênes (Sudbury, Prise de parole, «Agora », 2005). Pour plus de détails, on consultera La Mouvée, bulletin d'information de la Cofram, $\mathrm{n}^{\circ} 2$, décembre 2006, 8 p., qui est disponible au site www.usainteanne.ca/ documents/la_mouvee_2.pdf.

JeAn-Pierre Pichette

\section{Chaire de recherche McCain en ethnologie acadienne (CREMEA)}

Département d'Études françaises

Université de Moncton

Téléphone : (506) 858-4592

Moncton (Nouveau-Brunswick) Courriel : ronald.labelle@umoncton.ca E1A 3E9

La Chaire de recherche McCain en ethnologie acadienne (CREMEA) a été créée en 2006 grâce à un don généreux de la Fondation McCain. Elle a comme mandat de participer à la formation des étudiants au département d'Études françaises en dispensant des cours sur l'ethnologie acadienne, de contribuer aux activités de recherche menées au sein du département, de former des équipes de chercheurs afin de faire avancer la recherche tant sur la culture acadienne traditionnelle que sur des phénomènes ethnologiques contemporains, de mettre en valeur les ressources patrimoniales acadiennes par la réalisation d'ouvrages destinés à un public varié et d'assurer une présence de l'Université de Moncton dans le domaine des études ethnologiques tant au Canada qu'à l'étranger.

\section{Programmes principaux}

La CREMEA a obtenu en mars 2007 une subvention de 29000 \$ du CRSH dans le cadre de son Programme de recherche et de diffusion lié aux langues officielles. Le projet s'intitule «Mise en valeur des ressources culturelles franco-terreneuviennes ». Ce projet vise à produire trois types de ressources documentaires : une trousse à vocation pédagogique comprenant des documents sonores sur disques compacts accompagnés de transcriptions, un recueil de chansons folkloriques accompagné d'illustrations sonores, et finalement un site électronique consacré à la culture traditionnelle francoterreneuvienne. 
Une autre subvention a été obtenue de la J. R. Smallwood Foundation afin de financer la transcription mélodique des chansons qui feront partie du recueil. Au cours de l'été 2007, deux étudiants effectuent des enquêtes sur le terrain et des recherches en archives à Terre-Neuve, alors qu'un musicien œuvre à la transcription mélodique. Tous ces travaux sont coordonnés par le titulaire de la chaire. Cette initiative constitue un projet pilote, car la CREMEA explorera la possibilité d'appliquer la formule à d'autres régions acadiennes.

Un second projet a été mis sur pied en partenariat avec la Helen Creighton Folklore Society d'Halifax. Intitulé « Chansons acadiennes de Pubnico et Chéticamp ", ce projet vise la publication d'une collection de chansons acadiennes recueillies par la pionnière des recherches ethnologiques en Nouvelle-Écosse, Helen Creighton. Le recueil sera accompagné d'information biographique sur les chanteurs, ainsi que d'un disque compact. La Helen Creighton Folklore Society investit $2000 \$$ dans le projet, auquel contribuent aussi la Fondation Helen-Creighton et la CREMEA.

Le titulaire de la CREMEA participe en tant que cochercheur au projet intitulé «Édition critique des œuvres fondamentales de la littérature acadienne », un projet dirigé par le professeur Denis Bourque au département d'Études françaises, et qui bénéficie d'une subvention importante du CRSH. Le titulaire a entrepris la préparation d'une édition critique de l'ouvrage intitulé L'École aux apparitions mystérieuses, paru en 1896.

Enfin, la CREMEA coordonne les activités d'un nouveau groupe de recherche à l'Université de Moncton, le « Groupe de recherche interdisciplinaire sur les traditions et la modernité » (GRITEM), composé au point de départ par les professeurs Ronald Labelle, Martin Geoffroy et Denise Lamontagne. Le GriTem bénéficie d'une subvention grâce à un programme spécial de financement des groupes de recherche à l'Université de Moncton. Le budget du Gritem, qui est administré par la CREMEA, permet aux membres de mener des recherches sur le terrain et facilitera aussi la tenue des journées d'étude permettant des échanges avec d'autres chercheurs. Ces activités aboutiront plus tard à la tenue d'un colloque à l'Université de Moncton sur le thème des traditions et la modernité.

\section{Activités d'enseignement}

Le titulaire a dispensé deux nouveaux cours au département d'Études françaises de l'Université de Moncton en 2006-2007. Le premier, « Ethnologie de l'Amérique française », fait un survol des études ethnologiques, alors que le second, «Légendes et récits de croyance », est un de trois nouveaux cours à caractère thématique offerts au département. À l'automne 2007, le titulaire est responsable du séminaire intitulé « Ethnolittérature acadienne », offert aux étudiants de $2^{\mathrm{e}}$ et de $3^{\mathrm{e}}$ cycle au département d'Études françaises. 


\section{Diffusion de la recherche}

Dans le cadre de la Journée internationale des musées en mai 2007, le titulaire a prononcé au Musée acadien de l'Université de Moncton une conférence intitulée « Le Patrimoine oral acadien : un trésor culturel universel». Dans le cadre de «La Résistance des marges », un colloque international qui se tient à l'Université Sainte-Anne en août 2007, le titulaire présente une conférence intitulée «Une charrette qui a traversé l'océan : les reflets de l'ankou breton dans la légende acadienne de la charrette de la mort ». Enfin, le titulaire participe avec l'ethnomusicologue Jeanette Gallant au colloque international « Acadiens et Cajuns : politique et culture de minorités ethniques en Amérique du Nord» tenu au Centre d'études canadiennes, Université d'Innsbruck, en septembre 2007. Leur communication conjointe s'intitule « La Place occupée par la chanson traditionnelle française dans la musique acadienne contemporaine : une adaptation difficile ».

\section{Services à la communauté universitaire}

En novembre 2006, le titulaire a rencontré M. Martin Fournier, le coordonnateur du projet d'Encyclopédie du patrimoine culturel de l'Amérique française, afin de discuter de la participation acadienne à ce projet. La CREMEA a depuis conseillé les responsables de l'Encyclopédie quant au contenu acadien et le titulaire a rédigé un texte intitulé « Le Tintamarre, une nouvelle "tradition" en Acadie ». Au début 2007, le titulaire a participé à une consultation menée par le doyen du « School of Arts and Community Studies » à l'Université du Cap-Breton concernant le projet d'établissement d'un programme de maîtrise en « Heritage Studies » au sein de cette institution. À l'Université de Moncton, le titulaire participe à la numérisation des collections des archives de folklore du Centre d'études acadiennes en tant que conseiller. La CREMEA finance présentement la numérisation de la collection Ronald Labelle et une collaboration a été établie avec le CÉA afin de faciliter la mise en application de nouvelles technologies dans le domaine des archives de folklore.

\section{Accueil de chercheurs}

La CREMEA a assisté divers chercheurs et étudiants pendant l'année 20062007, y compris plusieurs venus de l'extérieur pour consulter les ressources documentaires du Centre d'études acadiennes. Le titulaire a notamment accueilli Madame Patricia Heiniger-Casteret, ethnologue de l'Université de Pau en France, lors de sa mission de recherche en juin 2007. À l'été 2007, la CREMEA a aussi accueilli Michael Kœnig, un étudiant à la maîtrise à l'Université de Dresde en Allemagne, qui prépare une thèse sur la culture traditionnelle acadienne. 


\section{Services à la collectivité}

Pendant l'hiver 2007, le titulaire a présenté une chronique radiophonique hebdomadaire intitulée " Histoire d'une chanson » sur les ondes de RadioCanada Atlantique. Cette chronique avait pour but de présenter l'évolution de la chanson traditionnelle acadienne d'une façon abordable au grand public. En février 2007, le titulaire a aussi organisé une activité pédagogique entourant le conte traditionnel à l'école Champlain de Moncton. Il s'agit d'une initiative qui pourrait se poursuivre à l'avenir.

\section{Perspectives d'avenir}

Les perspectives de recherche qui s'offrent à la CREMEA sont nombreuses et prometteuses. D'abord, selon les résultats obtenus dans le projet « Mise en valeur des ressources culturelles franco-terreneuviennes », un modèle semblable pourrait être appliqué à plusieurs régions acadiennes. Le nouveau " Groupe de recherche interdisciplinaire sur les traditions et la modernité », qui vient d'entamer ses activités, promet aussi de stimuler de nouvelles recherches à l'Université de Moncton, où le domaine de l'ethnologie fait maintenant l'objet de travaux concertés. La relance des activités dans le domaine du folklore au Centre d'études acadiennes ouvre maintenant de nouvelles perspectives et des discussions ont déjà lieu entre la CREMEA et la direction du CÉA en vue de collaborer à la création de nouvelles ressources électroniques.

Au cours des prochaines années, la CREMEA aura une présence continue au sein de l'important projet « Édition critique des œuvres fondamentales de la littérature acadienne ». L'édition critique de L'École aux apparitions mystérieuses pourrait ainsi être suivie d'autres initiatives impliquant la Chaire. Dans le domaine du conte traditionnel, un projet important pourra être mis en place impliquant des ethnologues au Canada, en France et en Louisiane. Des discussions initiales ont déjà eu lieu avec des spécialistes dans le domaine et le projet pourrait prendre forme au cours de la prochaine année.

Plusieurs ethnologues consacrent leurs efforts au domaine émergent du patrimoine immatériel aujourd'hui et la CREMEA a de nombreux contacts avec les spécialistes de l'Université Laval, de l'Université du Cap-Breton, de l'Université Memorial de Terre-Neuve et de l'Université Sainte-Anne. Tous sont appelés à jouer un rôle dans le développement de ce domaine dont l'UNESCO fait aujourd'hui la promotion par la nouvelle Convention pour la sauvegarde du patrimoine culturel immatériel. À l'extérieur du milieu universitaire, les contacts avec des groupes comme « La Nuit internationale du conte en Acadie » (NICA) amènent aussi des collaborations fructueuses. À titre d'exemple, une figure de proue du renouveau du conte en France, Henri 
Gougaud, sera présent à l'Université de Moncton à l'automne, grâce à une collaboration avec la NiCA. Enfin, un site électronique consacré au CREMEA et à ses activités verra le jour pendant l'année 2007-2008, ce qui permettra d'informer les intéressés aux activités de la chaire d'une façon continue.

RonALD LABELLE 